\title{
A Calderon Multiplicative Preconditioner for the PMCHWT Equation for Scattering by Chiral Objects
}

\author{
Yves Beghein, Kristof Cools, Francesco P. Andriulli, Daniël De Zutter, Eric Michielssen
}

\begin{abstract}
Scattering of time-harmonic electromagnetic waves by chiral structures can be modeled via an extension of the PMCHWT boundary integral equation for analyzing scattering by dielectric objects. The classical PMCHWT equation however suffers from dense discretization breakdown: the matrices resulting from its discretization become increasingly ill-conditioned when the mesh density increases. This contribution revisits the PMCHWT equation for chiral media. It is demonstrated that it also suffers from dense discretization breakdown. This dense discretization breakdown is mitigated by the construction of a Calderón multiplicative preconditioner. A stable discretization scheme is introduced, and the resulting algorithm's accuracy and efficiency are corroborated by numerical examples.
\end{abstract}

Index Terms-chiral media, boundary integral equations, boundary element method, PMCHWT equation, dense discretization breakdown, Calderón multiplicative preconditioner

\section{INTRODUCTION}

$\mathbf{S}$ CATTERING of time-harmonic electromagnetic fields by homogeneous objects often is modeled using boundary integral equations (BIEs). The best-known BIEs that apply to scattering by perfect electric conductors are the electric and magnetic field integral equations (EFIE and MFIE). Prototypical BIEs that apply to penetrable objects are the PoggioMiller-Chan-Harrington-Wu-Tsai (PMCHWT) [1] and Müller equations [2]. These equations can be regarded as analogues of the EFIE and MFIE, in that they exhibit similar spectral properties. More precisely, the spectra of the EFIE's and the PMCHWT equation's operators comprise two branches, one accumulating at zero, and the other at infinity. The spectra of the MFIE's and the Müller equation's operators, on the other hand, accumulate at a finite non-zero value.

All of the above BIEs can be solved numerically using the boundary element method: the surface of the scatterer is approximated by a mesh, and the unknown field quantities are expanded in a finite number of basis functions defined on this mesh. This approach reduces the BIE to a finite set

Y. Beghein and D. De Zutter are with the Department of Information Technology, Ghent University, Ghent 9000, Belgium (e-mail: yves.beghein@ugent.be).

K. Cools is with the Electrical Systems and Optics Research Division, University of Nottingham, Nottingham NG7 2RD, UK.

F. P. Andriulli is with the Microwave Department, École Nationale Supérieure des Télécommunications de Bretagne (TELECOM Bretagne), Brest 29238, France.

E. Michielssen is with the Department of Electrical Engineering and Computer Science, University of Michigan, Ann Arbor, MI 48109 USA. His efforts on this project were funded by AFOSR/NSSEFF Program Award FA9550-10-1-0180. of linear equations in the field expansion coefficients, which often is solved using iterative methods. Because the EFIE's and PMCHWT equation's operators have unbounded spectra, the corresponding system matrices have very large condition numbers when the mesh parameter (i.e. the length of the shortest edge) decreases. This phenomenon is called dense discretization breakdown and can be resolved by applying Calderón multiplicative preconditioners (CMPs) [3]-[5].

In [6], the authors have presented the successful application of the CMP to the chiral extension of the PMCHWT equation. It is the aim of this paper to elaborate on the theoretical fundamentals of the chiral CMP, and to present further numerical experiments corroborating the accuracy and the efficiency of the technique.

Section II offers a quick overview of electromagnetic fields in chiral media. Following this, the PMCHWT equation for chiral media is presented in section III. While this is not new material, it is included for self-containedness and to introduce the notations used throughout the following sections.

In section IV, the self-regularizing property of the chiral PMCHWT operator is studied. While this is analogous to the nonchiral case, two difficulties arise: the spectral properties of a composite operator involving three different wavenumbers must be studied, and the chiral PMCHWT operator requires a diagonalization. Once it is established that the operator is indeed self-regularizing, the CMP is formed by applying a suitable discretization scheme.

In the last section, numerical experiments testify to the success of the CMP. The accuracy is tested by comparing the results to the Mie series and to ab initio simulations of chiral metamaterials. The efficiency is shown by comparing the condition number of the system matrix with and without CMP, and the required number of iterations.

\section{Electromagnetic Fields in Chiral Media}

For time-harmonic electromagnetic fields, the electric and magnetic field (resp. $\boldsymbol{e}$ and $\boldsymbol{h}$ ) are linked to the electric and magnetic displacement field (resp. $\boldsymbol{d}$ and $\boldsymbol{b}$ ) by the Maxwell equations

$$
\begin{aligned}
\nabla \times \boldsymbol{e} & =-j \omega \boldsymbol{b}-\boldsymbol{j}_{m} \\
\nabla \times \boldsymbol{h} & =j \omega \boldsymbol{d}+\boldsymbol{j},
\end{aligned}
$$

where $\omega=2 \pi f$ is the angular frequency, $\boldsymbol{j}$ is the electric current density, and $\boldsymbol{j}_{m}$ is the magnetic current density. 
The interaction of the electromagnetic fields with the medium in which they propagate is modeled by constitutive equations. The lack of reflection symmetry, which is the defining characteristic for chiral media, must therefore be incorporated into the constitutive equations. This can be done using the Drude-Born-Fedorov (DBF) model [7]. However, for notational simplicity, the following equivalent constitutive equations are assumed [8]:

$$
\left(\begin{array}{l}
\boldsymbol{d} \\
\boldsymbol{b}
\end{array}\right)=\left(\begin{array}{cc}
\epsilon & -j \kappa \sqrt{\epsilon \mu} \\
j \kappa \sqrt{\epsilon \mu} & \mu
\end{array}\right)\left(\begin{array}{l}
\boldsymbol{e} \\
\boldsymbol{h}
\end{array}\right),
$$

where $\epsilon$ is the permittivity, $\mu$ is the permeability and $\kappa$ is the dimensionless chirality parameter. The constitutive equations (3) introduce extra coupling between electric and magnetic field quantities. This coupling can be removed, however, by application of the so-called Bohren transform:

$$
\left(\begin{array}{c}
\mathcal{E}_{ \pm} \\
\mathcal{H}_{ \pm}
\end{array}\right)=P^{ \pm}\left(\begin{array}{l}
\boldsymbol{e} \\
\boldsymbol{h}
\end{array}\right), \quad P^{ \pm}=\frac{1}{2}\left(\begin{array}{cc}
1 & \mp j \eta \\
\pm \frac{j}{\eta} & 1
\end{array}\right)
$$

Here $\eta=\sqrt{\mu / \epsilon}$ is the characteristic impedance of the medium. The matrices $P^{ \pm}$are projection matrices, satisfying $P^{+}+P^{-}=1$. The inverse Bohren transform is

$$
\boldsymbol{e}=\mathcal{E}_{+}+\mathcal{E}_{-}, \quad \boldsymbol{h}=\mathcal{H}_{+}+\mathcal{H}_{-} .
$$

A similar transformation applies to the electric and magnetic sources:

$$
\left(\begin{array}{c}
\mathcal{J}_{ \pm} \\
\mathcal{J}_{m, \pm}
\end{array}\right)=\frac{1}{2}\left(\begin{array}{cc}
1 & \mp \frac{j}{\eta} \\
\pm j \eta & 1
\end{array}\right)\left(\begin{array}{c}
\boldsymbol{j} \\
\boldsymbol{j}_{m}
\end{array}\right)
$$

The fields resulting from the Bohren transforms (4) and (6) are governed by equations formally identical to Maxwell equations in the absence of chirality:

$$
\begin{aligned}
\nabla \times \mathcal{E}_{ \pm} & =-j \omega \mu(1 \pm \kappa) \mathcal{H}_{ \pm}-\mathcal{J}_{m, \pm} \\
\nabla \times \mathcal{H}_{ \pm} & =j \omega \epsilon(1 \pm \kappa) \mathcal{E}_{ \pm}+\mathcal{J}_{ \pm} .
\end{aligned}
$$

Equation (4) decomposes the electromagnetic fields $(\boldsymbol{e}, \boldsymbol{h})$ into two contributions: $\left(\mathcal{E}_{+}, \mathcal{H}_{+}\right)$and $\left(\mathcal{E}_{-}, \mathcal{H}_{-}\right)$. In the absence of external sources,

$$
\begin{aligned}
\nabla \times \mathcal{E}_{ \pm} & = \pm \gamma_{ \pm} \mathcal{E}_{ \pm} \\
\gamma_{ \pm} & =\omega \sqrt{\epsilon \mu}(1 \pm \kappa) .
\end{aligned}
$$

Therefore, $\mathcal{E}_{+}$and $\mathcal{E}_{-}$are Beltrami fields. For $-1<\kappa<1$ (which is the case for realistic materials), the two "modes" $\mathcal{E}_{+}$and $\mathcal{E}_{-}$have opposite helicity, and the operators $P^{+}$and $P^{-}$project the electromagnetic fields upon their components with positive and negative helicity, respectively. When applied to plane wave solutions, the component with positive helicity $\left(\mathcal{E}_{+}, \mathcal{H}_{+}\right)$is right-handed circularly polarized, while the component with negative helicity $\left(\mathcal{E}_{-}, \mathcal{H}_{-}\right)$is left-handed circularly polarized. If the chirality parameter $\kappa$ is nonzero, these components propagate with different wavenumbers $\gamma_{ \pm}$. This is the cause of the well-known phenomenon of optical activity.

\section{The ChIRAL PMCHWT EQUATION}

The Bohren transform allows for the expansion of the electromagnetic field in a chiral medium into two components, each fulfilling the Maxwell equations in a nonchiral medium. These components thus obey all the usual equations and identities of electromagnetics. This property can be exploited to construct BIEs describing scattering by chiral objects. In this section, the extension of the PMCHWT equation for the modeling of scattering by nonchiral objects to the chiral PMCHWT equation for the modeling of scattering by chiral structures is revisited.

\section{A. The Stratton-Chu Representation Theorem}

Consider a homogeneous, isotropic, penetrable object $\Omega$ characterized by permittivity $\epsilon$, permeability $\mu$, impedance $\eta=\sqrt{\mu / \epsilon}$ and wavenumber $k=\omega \sqrt{\mu \epsilon}$. It is embedded in a background medium with permittivity $\epsilon_{0}$, permeability $\mu_{0}$, impedance $\eta_{0}=\sqrt{\mu_{0} / \epsilon_{0}}$ and wavenumber $k_{0}=\omega \sqrt{\mu_{0} \epsilon_{0}}$. The boundary of $\Omega$ is denoted $\Gamma$, and its exterior normal vector is denoted $\hat{\boldsymbol{n}}$. An external electromagnetic field $\left(\boldsymbol{e}^{i}, \boldsymbol{h}^{i}\right)$ is applied. The tangential traces of the electromagnetic field just inside $\Omega$, denoted $\left(\boldsymbol{e}^{-}, \boldsymbol{h}^{-}\right)$, satisfy the Stratton-Chu representation formula [9]:

$$
\left(\begin{array}{c}
-\hat{\boldsymbol{n}} \times \boldsymbol{e}^{-} \\
\hat{\boldsymbol{n}} \times \boldsymbol{h}^{-}
\end{array}\right)=\mathcal{S}^{\mathrm{int}}(\eta, k)\left(\begin{array}{c}
-\hat{\boldsymbol{n}} \times \boldsymbol{e}^{-} \\
\hat{\boldsymbol{n}} \times \boldsymbol{h}^{-}
\end{array}\right),
$$

where

$$
\mathcal{S}^{\text {int }}(\eta, k)=\left(\begin{array}{cc}
\frac{1}{2}-\mathcal{K}_{k} & \eta \mathcal{T}_{k} \\
-\mathcal{T}_{k} / \eta & \frac{1}{2}-\mathcal{K}_{k}
\end{array}\right)
$$

is the internal Stratton-Chu operator. A similar result holds for the traces of the electromagnetic field just outside $\Omega$, denoted $\left(e^{+}, h^{+}\right):$

$$
\left(\begin{array}{c}
-\hat{\boldsymbol{n}} \times \boldsymbol{e}^{+} \\
\hat{\boldsymbol{n}} \times \boldsymbol{h}^{+}
\end{array}\right)=\mathcal{S}^{\operatorname{ext}}\left(\eta_{0}, k_{0}\right)\left(\begin{array}{c}
-\hat{\boldsymbol{n}} \times \boldsymbol{e}^{+} \\
\hat{\boldsymbol{n}} \times \boldsymbol{h}^{+}
\end{array}\right)+\left(\begin{array}{c}
-\hat{\boldsymbol{n}} \times \boldsymbol{e}^{i} \\
\hat{\boldsymbol{n}} \times \boldsymbol{h}^{i}
\end{array}\right),
$$

where

$$
\mathcal{S}^{\text {ext }}\left(\eta_{0}, k_{0}\right)=\left(\begin{array}{ll}
\frac{1}{2}+\mathcal{K}_{k_{0}} & -\eta_{0} \mathcal{T}_{k_{0}} \\
\mathcal{T}_{k_{0}} / \eta_{0} & \frac{1}{2}+\mathcal{K}_{k_{0}}
\end{array}\right)
$$

is the external Stratton-Chu operator. The EFIE operator $\mathcal{T}_{k}$ (which is the sum of a weakly singular part $\mathcal{T}_{s}$ and a hypersingular part $\mathcal{T}_{h}$ ) and the MFIE operator $\mathcal{K}_{k}$ are

$$
\begin{aligned}
\mathcal{T}_{k} \boldsymbol{f}(\boldsymbol{r}) & =\mathcal{T}_{S, k} \boldsymbol{f}(\boldsymbol{r})+\mathcal{T}_{H, k} \boldsymbol{f}(\boldsymbol{r}), \\
\mathcal{T}_{S, k} \boldsymbol{f}(\boldsymbol{r}) & =-j k \hat{\boldsymbol{n}} \times \int_{\Gamma} \frac{e^{-j k R}}{4 \pi R} \boldsymbol{f}\left(\boldsymbol{r}^{\prime}\right) d \mathbf{s}^{\prime} \\
\mathcal{T}_{H, k} \boldsymbol{f}(\boldsymbol{r}) & =\frac{1}{j k} \hat{\boldsymbol{n}} \times p \cdot v \cdot \int_{\Gamma} \nabla \frac{e^{-j k R}}{4 \pi R} \nabla^{\prime} \cdot \boldsymbol{f}\left(\boldsymbol{r}^{\prime}\right) d \mathbf{s}^{\prime}(1) \\
\mathcal{K}_{k} \boldsymbol{f}(\boldsymbol{r}) & =\hat{\boldsymbol{n}} \times p . v \cdot \int_{\Gamma} \nabla \times \frac{e^{-j k R}}{4 \pi R} \boldsymbol{f}\left(\boldsymbol{r}^{\prime}\right) d \mathbf{s}^{\prime},
\end{aligned}
$$

where p.v. indicates that the integral should be interpreted as a Cauchy principal value, and $R=\left|\boldsymbol{r}-\boldsymbol{r}^{\prime}\right|$.

By imposing continuity of the tangential traces of $\left(\boldsymbol{e}^{-}, \boldsymbol{h}^{-}\right)$ and $\left(\boldsymbol{e}^{+}, \boldsymbol{h}^{+}\right)$, and subtracting (11) from (13), the PMCHWT equation [1] is obtained:

$$
\mathcal{Q}\left(\eta_{0}, k_{0} ; \eta, k\right)\left(\begin{array}{c}
-\hat{\boldsymbol{n}} \times \boldsymbol{e} \\
\hat{\boldsymbol{n}} \times \boldsymbol{h}
\end{array}\right)=-\left(\begin{array}{c}
-\hat{\boldsymbol{n}} \times \boldsymbol{e}^{i} \\
\hat{\boldsymbol{n}} \times \boldsymbol{h}^{i}
\end{array}\right)
$$


where the PMCHWT operator is

$$
\begin{aligned}
\mathcal{Q}\left(\eta_{0}, k_{0} ; \eta, k\right) & =\mathcal{S}^{\mathrm{ext}}\left(\eta_{0}, k_{0}\right)-\mathcal{S}^{\mathrm{int}}(\eta, k) \\
& =\left(\begin{array}{cc}
\mathcal{K}_{\mathrm{k}_{0}}+\mathcal{K}_{k} & -\eta_{0} \mathcal{T}_{\mathrm{k}_{0}}-\eta \mathcal{T}_{k} \\
\mathcal{T}_{\mathrm{k}_{0}} / \eta_{0}+\mathcal{T}_{k} / \eta & \mathcal{K}_{\mathrm{k}_{0}}+\mathcal{K}_{k}
\end{array}\right) .
\end{aligned}
$$

\section{B. The PMCHWT Equation for Chiral Media}

If the medium filling $\Omega$ is chiral, equation (11) does not hold. However, the Bohren transform has shown that in chiral media, the field comprises two components $\left(\mathcal{E}_{ \pm}, \mathcal{H}_{ \pm}\right)$that do not couple (except at boundaries). They act as though they propagate through a nonchiral medium with characteristic impedance $\eta$ and wavenumbers $\gamma_{ \pm}$. Therefore, they obey the Stratton-Chu representation formula (11):

$$
\left(\begin{array}{c}
-\hat{\boldsymbol{n}} \times \mathcal{E}_{ \pm} \\
\hat{\boldsymbol{n}} \times \mathcal{H}_{ \pm}
\end{array}\right)=\mathcal{S}^{\operatorname{int}}\left(\eta, \gamma_{ \pm}\right)\left(\begin{array}{c}
-\hat{\boldsymbol{n}} \times \mathcal{E}_{ \pm} \\
\hat{\boldsymbol{n}} \times \mathcal{H}_{ \pm}
\end{array}\right)
$$

Transforming this back to $e$ and $\boldsymbol{h}$ using (4) and (5) results in

$$
\begin{aligned}
\left(\begin{array}{c}
-\hat{\boldsymbol{n}} \times \boldsymbol{e}^{-} \\
\hat{\boldsymbol{n}} \times \boldsymbol{h}^{-}
\end{array}\right) & =\mathcal{S}_{c}^{\text {int }}\left(\eta, \gamma_{-}, \gamma_{+}\right)\left(\begin{array}{c}
-\hat{\boldsymbol{n}} \times \boldsymbol{e}^{-} \\
\hat{\boldsymbol{n}} \times \boldsymbol{h}^{-}
\end{array}\right), \\
\mathcal{S}_{c}^{\text {int }}\left(\eta, \gamma_{-}, \gamma_{+}\right) & =\mathcal{S}^{\text {int }}\left(\eta, \gamma_{+}\right) P^{-}+\mathcal{S}^{\text {int }}\left(\eta, \gamma_{-}\right) P^{+} .
\end{aligned}
$$

The pairing of $\gamma_{+}$with $P^{-}$and $\gamma_{-}$with $P^{+}$stems from

$$
\left(\begin{array}{c}
\mathcal{E}^{ \pm} \\
\mathcal{H}^{ \pm}
\end{array}\right)=P^{ \pm}\left(\begin{array}{c}
\boldsymbol{e} \\
\boldsymbol{h}
\end{array}\right) \Rightarrow\left(\begin{array}{c}
-\hat{\boldsymbol{n}} \times \mathcal{E}^{ \pm} \\
\hat{\boldsymbol{n}} \times \mathcal{H}^{ \pm}
\end{array}\right)=P^{\mp}\left(\begin{array}{c}
-\hat{\boldsymbol{n}} \times \boldsymbol{e} \\
\hat{\boldsymbol{n}} \times \boldsymbol{h}
\end{array}\right) .
$$

The chiral PMCHWT equation is obtained by again imposing continuity of the tangential traces of $\left(\boldsymbol{e}^{-}, \boldsymbol{h}^{-}\right)$and $\left(\boldsymbol{e}^{+}, \boldsymbol{h}^{+}\right)$, and subtracting (13) from (22):

$$
\mathcal{Q}_{c}\left(\eta_{0}, k_{0} ; \eta, \gamma_{-}, \gamma_{+}\right)\left(\begin{array}{c}
-\hat{\boldsymbol{n}} \times \boldsymbol{e} \\
\hat{\boldsymbol{n}} \times \boldsymbol{h}
\end{array}\right)=-\left(\begin{array}{c}
-\hat{\boldsymbol{n}} \times \boldsymbol{e}^{i} \\
\hat{\boldsymbol{n}} \times \boldsymbol{h}^{i}
\end{array}\right)
$$

where the chiral PMCHWT operator is

$$
\begin{aligned}
& \mathcal{Q}_{c}\left(\eta_{0}, k_{0} ; \eta, \gamma_{-}, \gamma_{+}\right) \\
= & \mathcal{S}^{\mathrm{ext}}\left(\eta_{0}, k_{0}\right)-\mathcal{S}^{\text {int }}\left(\eta, \gamma_{+}\right) P^{-}-\mathcal{S}^{\text {int }}\left(\eta, \gamma_{-}\right) P^{+} \\
= & \mathcal{Q}\left(\eta_{0}, k_{0} ; \eta, \gamma_{+}\right) P^{-}+\mathcal{Q}\left(\eta_{0}, k_{0} ; \eta, \gamma_{-}\right) P^{+} \\
= & \left(\begin{array}{ll}
\mathcal{Q}_{11} & \mathcal{Q}_{12} \\
\mathcal{Q}_{21} & \mathcal{Q}_{22}
\end{array}\right) .
\end{aligned}
$$

Expressions for the operators $\mathcal{Q}_{11}, \mathcal{Q}_{12}, \mathcal{Q}_{21}$ and $\mathcal{Q}_{22}$ are obtained by combining (4), (12) and (14):

$$
\begin{aligned}
\mathcal{Q}_{11}=\mathcal{Q}_{22} & =\mathcal{K}_{\mathrm{k}_{0}}+\mathcal{K}^{+}-j \mathcal{T}^{-}, \\
\mathcal{Q}_{12} & =-\eta_{0} \mathcal{T}_{\mathrm{k}_{0}}-\eta\left(\mathcal{T}^{+}+j \mathcal{K}^{-}\right), \\
\mathcal{Q}_{21} & =\mathcal{T}_{\mathrm{k}_{0}} / \eta_{0}+\left(\mathcal{T}^{+}+j \mathcal{K}^{-}\right) / \eta
\end{aligned}
$$

Here the notation

$$
\mathcal{K}^{ \pm}=\frac{1}{2}\left(\mathcal{K}_{\gamma_{-}} \pm \mathcal{K}_{\gamma_{+}}\right) \quad, \quad \mathcal{T}^{ \pm}=\frac{1}{2}\left(\mathcal{T}_{\gamma_{-}} \pm \mathcal{T}_{\gamma_{+}}\right)
$$

was used.

The chiral PMCHWT equation is obtained by performing the following substitution in the nonchiral PMCHWT equation:

$$
\mathcal{K}_{k} \rightarrow \mathcal{K}^{+}-j \mathcal{T}^{-} \quad, \quad \mathcal{T}_{k} \rightarrow \mathcal{T}^{+}+j \mathcal{K}^{-}
$$

Note that the compact contributions $\mathcal{K}_{k}$ from the nonchiral PMCHWT equation are perturbed by discontinuous contributions $j \mathcal{T}^{-}$. This implies that no matter how small the chirality parameter $\kappa$, there always is a mesh parameter $h_{0}$ such that when the actual mesh parameter $h$ is smaller than $h_{0}$, the spectra of the matrices resulting upon discretization of the nonchiral and chiral PMCHWT equation will differ qualitatively. This is symptomatic to the introduction of "new physics" in the system.

The chiral PMCHWT equation (24) is equivalent to that presented in [10], and is a special case of the integral equations constructed for inhomogeneous chiral structures in [11]. A similar boundary integral equation has been derived for scattering by chiral objects above a lossy half space [12], [13]. The chiral PMCHWT equation can also be applied to chiral scatterers in chiral background media [14].

\section{Numerical Solution of the Chiral PMCHWT equation}

To solve (24) via the boundary element method, the unknown quantities $-\hat{\boldsymbol{n}} \times \boldsymbol{e}$ and $\hat{\boldsymbol{n}} \times \boldsymbol{h}$ are expanded in a set of basis functions $\boldsymbol{f}_{i}$ :

$$
-\hat{\boldsymbol{n}} \times \boldsymbol{e}=\sum_{i=1}^{N} \mathrm{c}_{i} \boldsymbol{f}_{i} \quad, \quad \hat{\boldsymbol{n}} \times \boldsymbol{h}=\sum_{i=1}^{N} \mathrm{~d}_{i} \boldsymbol{f}_{i} .
$$

These expansions are inserted into (24), and the resulting equations are tested with a set of testing functions $\tilde{\boldsymbol{f}}_{i}$ (i.e. multiplied by $\tilde{f}_{i}$ and integrated over $\Gamma$ ). This results in the following set of linear equations (in matrix form):

$$
\left(\begin{array}{ll}
\mathbf{Q}_{11} & \mathbf{Q}_{12} \\
\mathbf{Q}_{21} & \mathbf{Q}_{22}
\end{array}\right)\left(\begin{array}{l}
\mathrm{c} \\
\mathrm{d}
\end{array}\right)=\left(\begin{array}{c}
\mathrm{e}^{i} \\
-\mathrm{h}^{i}
\end{array}\right)
$$

with

$$
\begin{aligned}
\left(\mathbf{Q}_{i j}\right)_{m n} & =\left(\tilde{\boldsymbol{f}}_{m}, \mathcal{Q}_{i j} \boldsymbol{f}_{n}\right), \\
\mathrm{e}_{m}^{i} & =\left(\tilde{\boldsymbol{f}}_{m}, \hat{\boldsymbol{n}} \times \boldsymbol{e}^{i}\right), \\
\mathrm{h}_{m}^{i} & =\left(\tilde{\boldsymbol{f}}_{m}, \hat{\boldsymbol{n}} \times \boldsymbol{h}^{i}\right), \\
(\boldsymbol{x}, \boldsymbol{y}) & =\int_{\Gamma} \boldsymbol{x}(\boldsymbol{r}) \cdot \boldsymbol{y}(\boldsymbol{r}) d s .
\end{aligned}
$$

This set of $2 N$ linear equations can be solved using a Krylov iterative solver.

The accuracy of the solution obtained by the boundary element method depends upon the spectral properties of the PMCHWT operator, as well as the choice of expansion and testing functions [15]. In our implementation, following standard practice [3], [4], the surface $\Gamma$ is approximated by a triangle mesh. The expansion functions $f_{i}$ are chosen to be div-conforming RWG functions [16], while the testing functions $\tilde{\boldsymbol{f}}_{i}$ are chosen to be curl-conforming rotated RWG functions $\hat{\boldsymbol{n}} \times \boldsymbol{f}_{i}$. The accuracy of the solution (27) then depends upon the density of the triangle mesh, which is measured by the mesh parameter (i.e. the minimum edge length). 


\section{The Calderón Multiplicative Preconditioner}

\section{A. Dense Discretization Breakdown}

The numerical solution of the EFIE suffers from dense discretization breakdown: when the mesh is made denser, the condition number of the system matrix in (28) grows quadratically as a function of the inverse of the mesh parameter. This renders the iterative solution of the discretized EFIE increasingly hard and time consuming.

The cause of this phenomenon is rooted in the mathematical properties of the EFIE operator. Its spectrum comprises two branches: one accumulating at zero, the other at infinity. As the discretization is made denser, eigenfunctions corresponding to eigenvalues accumulating at zero and infinity both can be resolved. This renders the system matrix ill-conditioned, and the iterative solution inefficient.

As the nonchiral PMCHWT operator (20) contains the EFIE operator as one of its constituents, it is not surprising that it too is susceptible to dense discretization breakdown [4].

Equation (25) indicates that the chiral PMCHWT operator is intimately connected with the nonchiral PMCHWT operator. Its spectrum can therefore also be expected to be unbounded, resulting in dense discretization breakdown.

To mitigate this problem, the EFIE and the PMCHWT equation have been regularized by Calderón multiplicative preconditioners (CMPs) [4], [5], [17]. In the next sections, this regularization procedure is elucidated and extended to the chiral PMCHWT equation.

\section{B. Regularizing the EFIE}

Dense discretization breakdown of the EFIE is caused by the unbounded spectrum of the EFIE operator $\mathcal{T}_{k}$. However, it is known that for smooth surfaces $\Gamma$, the eigenvalues of the MFIE operator $\mathcal{K}_{k}$ accumulate at zero (i.e. the operator is compact) [18]. The Calderón identities

$$
\begin{aligned}
\mathcal{K}_{\mathrm{k}}^{2}-\mathcal{T}_{\mathrm{k}}^{2} & =\frac{1}{4}, \\
\mathcal{T}_{\mathrm{k}} \mathcal{K}_{\mathrm{k}}+\mathcal{K}_{\mathrm{k}} \mathcal{T}_{\mathrm{k}} & =0
\end{aligned}
$$

imply that the EFIE operator is self-regularizing: the eigenvalues of its square accumulate at $-\frac{1}{4}$, and its spectrum therefore is bounded. Moreover, if the scatterer does not support an internal resonance at the wave number $k$, the spectrum is bounded away from zero. Upon discretization, such an operator results in a well-conditioned set of equations, even when the discretization is made denser. This fact inspired the introduction of the CMP EFIE in [17]:

$$
\eta \mathcal{T}_{k}^{2}(\boldsymbol{j})=-\mathcal{T}_{k}\left(\hat{\boldsymbol{n}} \times \boldsymbol{e}^{i}\right) .
$$

In [3], it has been shown that this equation, which involves a product of two operators, can be discretized in a conforming and stable manner by leveraging both RWG and BC functions and the introduction of the corresponding inverse Gram matrix.

\section{Regularizing the Nonchiral PMCHWT Equation}

In [4], it has been shown that the PMCHWT equation's operator too exhibits a self-regularizing property. In particular, it has been show that the CMP PMCHWT equation

$$
\mathcal{Q}\left(\eta_{0}, k_{0} ; \eta, k\right)^{2}\left(\begin{array}{c}
-\hat{\boldsymbol{n}} \times \boldsymbol{e} \\
\hat{\boldsymbol{n}} \times \boldsymbol{h}
\end{array}\right)=-\mathcal{Q}\left(\eta_{0}, k_{0} ; \eta, k\right)\left(\begin{array}{c}
-\hat{\boldsymbol{n}} \times \boldsymbol{e}^{i} \\
\hat{\boldsymbol{n}} \times \boldsymbol{h}^{i}
\end{array}\right)
$$

involves an operator whose spectrum accumulates at finite non-zero values. In addition to the Calderón identities, the proof requires the determination of the accumulation points of the spectra of the following two-wavenumber operators:

$$
\begin{aligned}
\mathcal{T}_{k_{1}} \mathcal{T}_{k_{2}} & \rightarrow-\frac{k_{1}}{4 k_{2}} \text { and }-\frac{k_{2}}{4 k_{1}}, \\
\mathcal{T}_{k_{2}} \mathcal{K}_{k_{1}}+\mathcal{K}_{k_{1}} \mathcal{T}_{k_{2}} & \rightarrow 0 .
\end{aligned}
$$

For $k_{1}=k_{2}$, this simply follows from the Calderón identities (29), (30). Ignoring all compact operators, it is then found that

$$
\mathcal{Q}\left(\eta_{0}, k_{0} ; \eta, k\right)^{2} \rightarrow\left(\frac{1}{2}-\frac{\eta}{\eta_{0}} \mathcal{T}_{k_{0}} \mathcal{T}_{k}-\frac{\eta_{0}}{\eta} \mathcal{T}_{k} \mathcal{T}_{k_{0}}\right)\left(\begin{array}{ll}
1 & 0 \\
0 & 1
\end{array}\right)
$$

where the arrow " $\rightarrow$ " must be understood as an equality modulo compact contribution. This shows that the eigenvalues of the squared nonchiral PMCHWT operator will accumulate at finite nonzero values. Thus, upon discretization, the system matrix will be well-conditioned, uniformly with regard to the mesh parameter.

\section{Regularizing the Chiral PMCHWT Equation}

The results in [3], [4] suggest the introduction of the following CMP for the chiral PMCHWT equation (24):

$$
\begin{gathered}
\mathcal{Q}_{c}\left(\eta_{0}, k_{0} ; \eta, \gamma_{-}, \gamma_{+}\right)^{2}\left(\begin{array}{c}
-\hat{\boldsymbol{n}} \times \boldsymbol{e} \\
\hat{\boldsymbol{n}} \times \boldsymbol{h}
\end{array}\right) \\
=-\mathcal{Q}_{c}\left(\eta_{0}, k_{0} ; \eta, \gamma_{-}, \gamma_{+}\right)\left(\begin{array}{c}
-\hat{\boldsymbol{n}} \times \boldsymbol{e}^{i} \\
\hat{\boldsymbol{n}} \times \boldsymbol{h}^{i}
\end{array}\right) .
\end{gathered}
$$

The occurrence of a nonzero chirality parameter however complicates the spectral analysis. Two difficulties arise:

- Explicit computation of the operators in the operator block matrix $\mathcal{Q}_{c}^{2}$ reveals the presence of a new type of operator: the three-wavenumber operator $\mathcal{T}_{k_{0}} \mathcal{K}^{-}-\mathcal{K}^{-} \mathcal{T}_{k_{0}}$. To characterize the spectrum of $\mathcal{Q}_{c}^{2}$, the spectrum of this three-wavenumber operator needs to be understood. In section IV-D1, it will be shown that the threewavenumber operator is compact. This operator therefore does not qualitatively affect the spectrum of $\mathcal{Q}_{c}^{2}$.

- The explicit expression of $\mathcal{Q}_{c}^{2}$ contains noncompact contributions in both the on- and off-diagonal blocks, complicating the study of the spectrum of the operator as a whole. To alleviate this difficulty, a suitable diagonalizing transformation (up to compact contributions) will be introduced in section IV-D2.

1) Spectrum of the three-wavenumber operator: Compactness of the three-wavenumber operator $\mathcal{T}_{k_{0}} \mathcal{K}^{-}-\mathcal{K}^{-} \mathcal{T}_{k_{0}}$ can be demonstrated for spherical scatterers of unit radius. It is known that the operators $\mathcal{T}_{k}$ and $\mathcal{K}_{k}$ are (skew-)diagonal in the basis of vector spherical harmonics $\boldsymbol{X}_{l m}=\operatorname{curl}_{S} Y_{l m}$ and $\boldsymbol{Y}_{l m}=\operatorname{grad}_{S} Y_{l m}$, with $\operatorname{curl}_{S}$ and $\operatorname{grad}_{S}$ the surface curl and 
surface gradient, respectively, and $Y_{l m}$ the spherical harmonics [17]:

$$
\begin{aligned}
\mathcal{T}_{k} \boldsymbol{X}_{l m} & =-\mathbb{J}_{l}(k) \mathbb{H}_{l}(k) \boldsymbol{Y}_{l m}, \\
\mathcal{T}_{k} \boldsymbol{Y}_{l m} & =\mathbb{J}_{l}^{\prime}(k) \mathbb{H}_{l}^{\prime}(k) \boldsymbol{X}_{l m}, \\
\mathcal{K}_{k} \boldsymbol{X}_{l m} & =\left(j \mathbb{J}_{l}^{\prime}(k) \mathbb{H}_{l}(k)-\frac{1}{2}\right) \boldsymbol{X}_{l m}, \\
\mathcal{K}_{k} \boldsymbol{Y}_{l m} & =-\left(j \mathbb{J}_{l}(k) \mathbb{H}_{l}^{\prime}(k)+\frac{1}{2}\right) \boldsymbol{Y}_{l m}
\end{aligned}
$$

where $\mathbb{J}_{l}(k)$ and $\mathbb{H}_{l}(k)$ are the Riccati Bessel and Hankel functions, respectively. They are related to the spherical Bessel function $j_{l}(k)$ and the spherical Hankel function of the first kind $h_{l}^{(1)}(k)$ as

$$
\begin{aligned}
\mathbb{J}_{l}(k) & =k j_{l}(k), \\
\mathbb{H}_{l}(k) & =k h_{l}^{(1)}(k) .
\end{aligned}
$$

Using (35), it is found that

$$
\left(\mathcal{T}_{k_{0}} \mathcal{K}^{-}-\mathcal{K}^{-} \mathcal{T}_{k_{0}}\right)\left(\begin{array}{l}
\boldsymbol{X}_{l m} \\
\boldsymbol{Y}_{l m}
\end{array}\right)=\left(\begin{array}{cc}
0 & a_{l m} \\
b_{l m} & 0
\end{array}\right)\left(\begin{array}{l}
\boldsymbol{X}_{l m} \\
\boldsymbol{Y}_{l m}
\end{array}\right)
$$

with

$$
\left(\begin{array}{c}
a_{l m} \\
b_{l m}
\end{array}\right)=-\frac{j}{2}\left(\mathbb{J}_{l}\left(\gamma_{1}\right) \mathbb{H}_{l}\left(\gamma_{1}\right)-\mathbb{J}_{l}\left(\gamma_{2}\right) \mathbb{H}_{l}\left(\gamma_{2}\right)\right)^{\prime}\left(\begin{array}{l}
\mathbb{J}_{l}\left(k_{0}\right) \mathbb{H}_{l}\left(k_{0}\right) \\
\mathbb{J}_{l}^{\prime}\left(k_{0}\right) \mathbb{H}_{l}^{\prime}\left(k_{0}\right)
\end{array}\right) .
$$

From (36), it follows that the eigenvalues of $\mathcal{T}_{k_{0}} \mathcal{K}^{-}-\mathcal{K}^{-} \mathcal{T}_{k_{0}}$ are $\pm \sqrt{a_{l m} b_{l m}}$. For large $l$, these eigenvalues tend to [19]

$$
\pm \sqrt{a_{l m} b_{l m}} \rightarrow \pm \frac{k^{2} \kappa}{4 l^{3}}+\mathcal{O}\left(l^{-4}\right)
$$

and hence accumulate at zero. The operator $\mathcal{T}_{k_{0}} \mathcal{K}^{-}-\mathcal{K}^{-} \mathcal{T}_{k_{0}}$ therefore is compact. This conclusion is not limited to spherical objects: the numerical examples at the end of this paper will show that it also holds for non-spherical ones.

2) Diagonalization of the squared PMCHWT operator: Non-compact operators appear in the off-diagonal elements of $\mathcal{Q}_{c}\left(\eta_{0}, k_{0} ; \eta, \gamma_{-}, \gamma_{+}\right)^{2}$, which makes it difficult to make conclusive statements regarding the behavior of its spectrum. This complication can be resolved by expressing the chiral CMP PMCHWT equation (34) in terms of the circularly polarized components of the electromagnetic fields outside the scatterer, $e_{1}$ and $e_{2}$, as

$$
\left(\begin{array}{l}
\boldsymbol{e} \\
\boldsymbol{h}
\end{array}\right)=\left(\begin{array}{cc}
1 & 1 \\
-\frac{j}{\eta_{0}} & \frac{j}{\eta_{0}}
\end{array}\right)\left(\begin{array}{l}
\boldsymbol{e}_{1} \\
\boldsymbol{e}_{2}
\end{array}\right) .
$$

(Note the difference between (38) and the Bohren transform (4): in (38) the vacuum impedance $\eta_{0}$ is used instead of the impedance of the chiral medium $\eta$.) Transformation (38) on the fields induces the following transformation on the traces:

$$
\left(\begin{array}{c}
-\hat{\boldsymbol{n}} \times \boldsymbol{e} \\
\hat{\boldsymbol{n}} \times \boldsymbol{h}
\end{array}\right)=-\left(\begin{array}{cc}
1 & 1 \\
\frac{j}{\eta_{0}} & -\frac{j}{\eta_{0}}
\end{array}\right)\left(\begin{array}{l}
\hat{\boldsymbol{n}} \times \boldsymbol{e}_{1} \\
\hat{\boldsymbol{n}} \times \boldsymbol{e}_{2}
\end{array}\right) .
$$

The chiral CMP PMCHWT equation (34) can therefore be expressed as

$$
\begin{aligned}
& \left(\begin{array}{cc}
1 & 1 \\
\frac{j}{\eta_{0}} & -\frac{j}{\eta_{0}}
\end{array}\right)^{-1} \mathcal{Q}_{c}^{2}\left(\begin{array}{cc}
1 & 1 \\
\frac{j}{\eta_{0}} & -\frac{j}{\eta_{0}}
\end{array}\right)\left(\begin{array}{l}
\hat{\boldsymbol{n}} \times \boldsymbol{e}_{1} \\
\hat{\boldsymbol{n}} \times \boldsymbol{e}_{2}
\end{array}\right) \\
= & -\left(\begin{array}{cc}
1 & 1 \\
\frac{j}{\eta_{0}} & -\frac{j}{\eta_{0}}
\end{array}\right)^{-1} \mathcal{Q}_{c}\left(\begin{array}{cc}
1 & 1 \\
\frac{j}{\eta_{0}} & -\frac{j}{\eta_{0}}
\end{array}\right)\left(\begin{array}{l}
\hat{\boldsymbol{n}} \times \boldsymbol{e}_{1}^{i} \\
\hat{\boldsymbol{n}} \times \boldsymbol{e}_{2}^{i}
\end{array}\right)
\end{aligned}
$$

TABLE I

OVERVIEW OF SPECTRAL ACCUMULATION POINTS

\begin{tabular}{c|c} 
Operator & Spectral accumulation points \\
\hline $\mathcal{T}_{k}^{2}$ & $-\frac{1}{4}$ \\
$\mathcal{T}_{k_{1}} \mathcal{T}_{k_{2}}$ & $-\frac{k_{1}}{4 k_{2}},-\frac{k_{2}}{4 k_{1}}$ \\
$\mathcal{K}_{k_{1}} \mathcal{K}_{k_{2}}$ & 0 \\
$\mathcal{T}_{k_{1}} \mathcal{K}_{k_{2}}+\mathcal{K}_{k_{2}} \mathcal{T}_{k_{1}}$ & 0 \\
$\mathcal{T}_{k_{0}} \mathcal{K}^{-}-\mathcal{K}^{-} \mathcal{T}_{k_{0}}$ & 0
\end{tabular}

where the dependence of $\mathcal{Q}_{c}$ on material parameters has been left out to simplify the notation.

From a physical point of view, it is clear that this reformulation cannot change the essential properties of the PMCHWT operator. From an algebraic point of view, the matrix

$$
\mathcal{R}=\left(\begin{array}{ll}
\mathcal{R}_{11} & \mathcal{R}_{12} \\
\mathcal{R}_{21} & \mathcal{R}_{22}
\end{array}\right)=\left(\begin{array}{cc}
1 & 1 \\
\frac{j}{\eta_{0}} & -\frac{j}{\eta_{0}}
\end{array}\right)^{-1} \mathcal{Q}_{c}^{2}\left(\begin{array}{cc}
1 & 1 \\
\frac{j}{\eta_{0}} & -\frac{j}{\eta_{0}}
\end{array}\right)
$$

is connected to $\mathcal{Q}_{c}^{2}$ by a similarity transformation, and therefore has an identical spectrum. Up to compact contributions (such as the three-wavenumber operator discussed in the previous subsection), it is found that

$$
\begin{aligned}
\mathcal{R}_{11} \rightarrow & \frac{1}{2}-\left(\frac{\eta_{0}}{\eta} \mathcal{T}_{k_{0}} \mathcal{T}^{+}+\frac{\eta}{\eta_{0}} \mathcal{T}^{+} \mathcal{T}_{k_{0}}\right) \\
& +\left(\mathcal{T}^{-} \mathcal{T}_{k_{0}}+\mathcal{T}_{k_{0}} \mathcal{T}^{-}\right), \\
\mathcal{R}_{12} \rightarrow & 0, \\
\mathcal{R}_{21} \rightarrow & 0, \\
\mathcal{R}_{22} \rightarrow & \frac{1}{2}-\left(\frac{\eta_{0}}{\eta} \mathcal{T}_{k_{0}} \mathcal{T}^{+}+\frac{\eta}{\eta_{0}} \mathcal{T}^{+} \mathcal{T}_{k_{0}}\right) \\
& -\left(\mathcal{T}^{-} \mathcal{T}_{k_{0}}+\mathcal{T}_{k_{0}} \mathcal{T}^{-}\right) .
\end{aligned}
$$

The eigenvalues of the diagonal elements accumulate at a finite number of finite nonzero values, while the off-diagonal elements are compact (for an overview of the accumulation points, see table I). Therefore, it can be concluded that the spectrum of $\mathcal{R}$, and thus the spectrum of the squared chiral PMCHWT operator, is bounded from above and below and thus allows an unconditionally stable discretization, which will be discussed in the next subsection.

\section{E. Stable Discretization}

The chiral CMP PMCHWT equation is formed by action of the block operator $\mathcal{Q}_{c}$ on the left and right hand sides of the chiral PMCHWT equation (24):

$$
\left(\begin{array}{ll}
\mathcal{Q}_{11} & \mathcal{Q}_{12} \\
\mathcal{Q}_{21} & \mathcal{Q}_{22}
\end{array}\right)^{2}\left(\begin{array}{c}
-\hat{\boldsymbol{n}} \times \boldsymbol{e} \\
\hat{\boldsymbol{n}} \times \boldsymbol{h}
\end{array}\right)=-\left(\begin{array}{ll}
\mathcal{Q}_{11} & \mathcal{Q}_{12} \\
\mathcal{Q}_{21} & \mathcal{Q}_{22}
\end{array}\right)\left(\begin{array}{c}
-\hat{\boldsymbol{n}} \times \boldsymbol{e}^{i} \\
\hat{\boldsymbol{n}} \times \boldsymbol{h}^{i}
\end{array}\right) .
$$

The squared PMCHWT operator can be discretized elegantly by introducing a second set of expansion functions $\boldsymbol{g}_{i}$ and testing functions $\tilde{\boldsymbol{g}}_{i}$. The following system is obtained (in block matrix form):

$$
\begin{aligned}
& \left(\begin{array}{ll}
\mathbf{Q}_{11}^{\prime} & \mathbf{Q}_{12}^{\prime} \\
\mathbf{Q}_{21}^{\prime} & \mathbf{Q}_{22}^{\prime}
\end{array}\right)\left(\begin{array}{cc}
\mathbf{G}^{-1} & 0 \\
0 & \mathbf{G}^{-1}
\end{array}\right)\left(\begin{array}{ll}
\mathbf{Q}_{11} & \mathbf{Q}_{12} \\
\mathbf{Q}_{21} & \mathbf{Q}_{22}
\end{array}\right)\left(\begin{array}{l}
\mathrm{c} \\
\mathrm{d}
\end{array}\right) \\
= & \left(\begin{array}{ll}
\mathbf{Q}^{\prime}{ }_{11} & \mathbf{Q}^{\prime}{ }_{12} \\
\mathbf{Q}^{\prime}{ }_{21} & \mathbf{Q}_{22}^{\prime}
\end{array}\right)\left(\begin{array}{cc}
\mathbf{G}^{-1} & 0 \\
0 & \mathbf{G}^{-1}
\end{array}\right)\left(\begin{array}{c}
\mathrm{e}^{i} \\
-\mathrm{h}^{i}
\end{array}\right)
\end{aligned}
$$


with

$$
\begin{aligned}
\left(\mathbf{Q}_{i j}\right)_{m n} & =\left(\tilde{\boldsymbol{f}}_{m}, \mathcal{Q}_{i j} \boldsymbol{f}_{n}\right), \\
\left(\mathbf{Q}_{i j}^{\prime}\right)_{m n} & =\left(\tilde{\boldsymbol{g}}_{m}, \mathcal{Q}_{i j} \boldsymbol{g}_{n}\right), \\
\mathbf{G}_{m n} & =\left(\tilde{\boldsymbol{f}_{m}}, \boldsymbol{g}_{n}\right) .
\end{aligned}
$$

$\mathbf{Q}_{i j}$ results from discretizing the operator with the first set of basis and testing functions, and $\mathbf{Q}_{i j}^{\prime}$ is obtained using the second set. The Gram matrix $\mathbf{G}$ relates the first set of testing functions to the second set of expansion functions. The matrix $\mathbf{Q}^{\prime} \mathbf{G}^{-1}$ acts as a multiplicative preconditioner.

In order to obtain accurate results, both sets of expansion functions must be div-conforming, and both sets of testing functions must be curl-conforming. Secondly, the Gram matrix $\mathbf{G}$ must be well-conditioned. Finally, the operators $\mathcal{Q}_{i j}$ must be well-tested in $\mathbf{Q}_{i j}$ as well as $\mathbf{Q}_{i j}^{\prime}$.

For a triangular mesh, a suitable choice was presented in [3] and [4]. There, $\mathbf{Q}$ is computed using divergence-conforming RWG functions $\boldsymbol{f}_{i}$ and curl-conforming rotated RWG functions $\hat{\boldsymbol{n}} \times \boldsymbol{f}_{i}$ [16]. $\mathbf{Q}^{\prime}$ is computed using div-conforming BuffaChristiansen (BC) functions $\boldsymbol{g}_{i}$ and curl-conforming rotated BC functions $\hat{\boldsymbol{n}} \times \boldsymbol{g}_{i}$ [20]. This discretization scheme will also be used in the numerical examples in the following section.

This discretization scheme can be extended to curvilinear [21] as well as higher-order triangular elements [22]. A general procedure not restricted to triangular meshes is described in [23].

In the next section, the beneficial properties of the CMP will be corroborated by numerical examples.

\section{NUMERICAL EXAMPLES}

\section{A. Scattering by a Chiral Sphere}

As presented in [6], the accuracy of the chiral CMP PMCHWT equation and the proposed discretization scheme can be tested by comparing numerical results to analytical solutions for scattering by a chiral sphere [24]. For example, consider a sphere with radius 1 meter and material parameters $\epsilon=2 \epsilon_{0}, \mu=\mu_{0}$, and $\kappa=0.5$. It is embedded in vacuum, and illuminated by a circularly polarized plane wave propagating along the $z$ axis with frequency $90 \mathrm{MHz}$ :

$$
\begin{aligned}
\boldsymbol{e}^{ \pm}(x, y, z) & =\boldsymbol{p} \exp \left(-j k_{0} z\right), \\
\boldsymbol{h}^{ \pm}(x, y, z) & = \pm \frac{j}{\eta_{0}} \boldsymbol{e}^{ \pm}(x, y, z), \\
\boldsymbol{p} & =\hat{\mathbf{1}}_{x} \mp j \hat{\mathbf{1}}_{y} .
\end{aligned}
$$

$\left(\boldsymbol{e}^{+}, \boldsymbol{h}^{+}\right)$corresponds to a right-hand circularly polarized wave, while $\left(\boldsymbol{e}^{-}, \boldsymbol{h}^{-}\right)$corresponds to a left-hand circularly polarized wave. The radar cross section (RCS) obtained using the chiral CMP PMCHWT method (with $N=1398$ RWG expansion functions) is compared to the results from the Mie series in Fig. 1, and seen to be in excellent agreement.

While the sphere is geometrically fully symmetrical, leftright symmetry in the scattered field is broken by the microscopic chiral structure of the material. This causes the sphere to react differently upon illumination by left- and right-handed circularly polarized waves. This asymmetry is only exhibited when $\kappa \neq 0$.

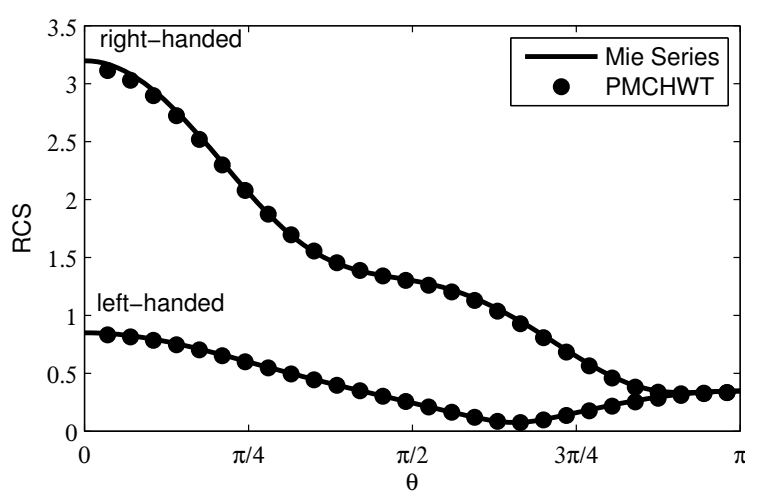

Fig. 1. Comparison of the chiral CMP PMCHWT method to the Mie series: the Radar Cross Section (RCS) in the $x z$ plane, with $\theta$ being the angle between the observed direction and the $z$ axis.

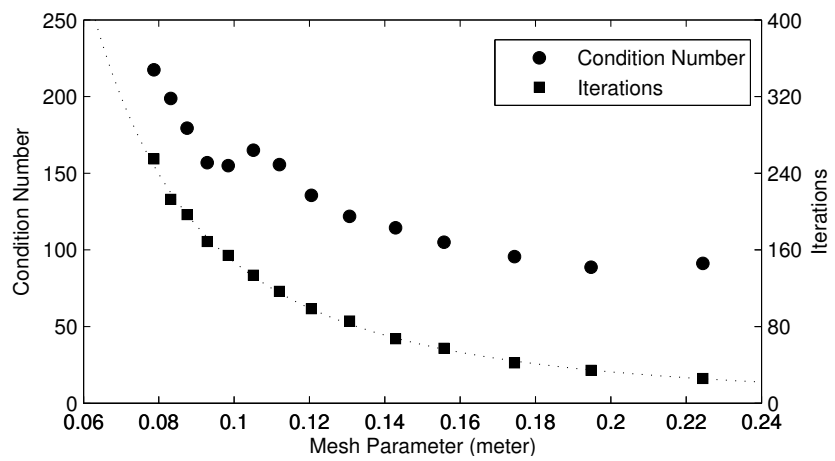

Fig. 2. The condition number of the system matrix and the number of iterations required to reach convergence without the CMP, as a function of the mesh parameter (in meters).

When solving the chiral PMCHWT method without the CMP, dense discretization breakdown occurs. To illustrate this, the condition number of the system matrix in (28) is plotted in Fig. 2 for increasingly dense discretizations alongside the number of iterations needed to reach convergence (arbitrarily defined as a relative residual smaller than $10^{-6}$ using the TFQMR method) when the sphere is illuminated by a linearly polarized plane wave.

However, when employing the CMP, the system matrix remains well-conditioned, no matter how small the mesh parameter. The condition number of the system matrix of (40) and the number of iterations needed to reach convergence for this scattering problem are shown in Fig. 3. It is clear that dense discretization breakdown is effectively cured by the CMP.

\section{B. Compactness of the three-wavenumber operator}

In section IV-D, we claimed that the three-wavenumber operator $\mathcal{T}_{k_{0}} \mathcal{K}^{-}-\mathcal{K}^{-} \mathcal{T}_{k_{0}}$ is compact, and proved this assertion for spherical scatterers. Now, the spectrum of this operator applied to a cube will be calculated by solving the eigenvalue equation

$$
\left(\mathcal{T}_{k_{0}} \mathcal{K}^{-}-\mathcal{K}^{-} \mathcal{T}_{k_{0}}\right) \boldsymbol{f}^{(\lambda)}=\lambda \boldsymbol{f}^{(\lambda)} .
$$




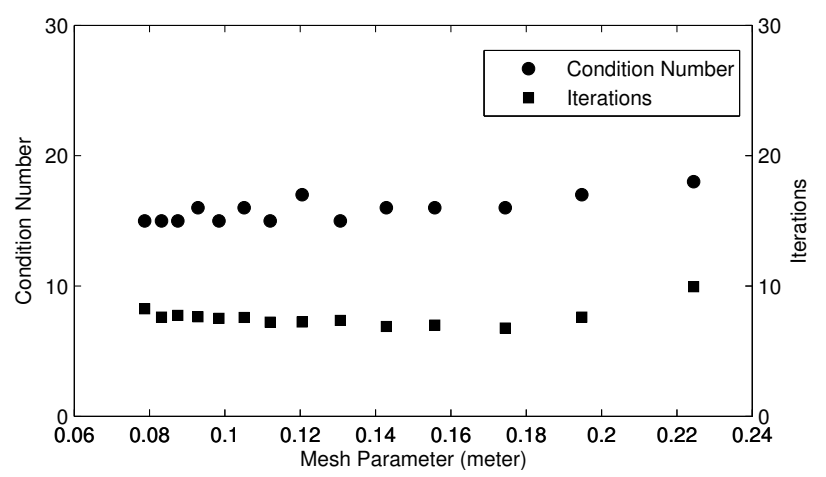

Fig. 3. The condition number of the system matrix and the number of iterations required to reach convergence with the CMP, as a function of the mesh parameter (in meters).

The eigenfunctions $\boldsymbol{f}^{(\lambda)}$ are approximated using RWG expansion functions:

$$
\boldsymbol{f}^{(\lambda)}=\sum_{i=1}^{N} \mathrm{a}_{i}^{(\lambda)} \boldsymbol{f}_{i} .
$$

By applying the discretization scheme used for the construction of the CMP, the eigenvalue equation (41) becomes

$$
\left(\mathbf{T}^{\prime}{ }_{k_{0}} \mathbf{G}^{-1} \mathbf{K}^{-}-\mathbf{K}^{\prime-} \mathbf{G}^{-1} \mathbf{T}_{k_{0}}\right) \mathbf{a}^{(\lambda)}=-\lambda \mathbf{G}^{T} \mathbf{a}^{(\lambda)}
$$

with

$$
\begin{aligned}
\left(\mathbf{T}_{k_{0}}\right)_{m n} & =\left(\hat{\boldsymbol{n}} \times \boldsymbol{f}_{m}, \mathcal{T}_{k_{0}} \boldsymbol{f}_{n}\right), \\
\left(\mathbf{T}^{\prime}{ }_{k_{0}}\right)_{m n} & =\left(\hat{\boldsymbol{n}} \times \boldsymbol{g}_{m}, \mathcal{T}_{k_{0}} \boldsymbol{g}_{n}\right), \\
\left(\mathbf{K}^{-}\right)_{m n} & =\left(\hat{\boldsymbol{n}} \times \boldsymbol{f}_{m}, \mathcal{K}^{-} \boldsymbol{f}_{n}\right), \\
\left(\mathbf{K}^{\prime-}\right)_{m n} & =\left(\hat{\boldsymbol{n}} \times \boldsymbol{g}_{m}, \mathcal{K}^{-} \boldsymbol{g}_{n}\right), \\
\mathbf{G}_{m n} & =\left(\hat{\boldsymbol{n}} \times \boldsymbol{f}_{n}, \boldsymbol{g}_{m}\right) .
\end{aligned}
$$

Thus, the spectrum of the operator $\mathcal{T}_{k_{0}} \mathcal{K}^{-}-\mathcal{K}^{-} \mathcal{T}_{k_{0}}$ is approximated by the spectrum of the matrix

$$
-\left(\mathbf{G}^{T}\right)^{-1}\left(\mathbf{T}^{\prime}{ }_{k_{0}} \mathbf{G}^{-1} \mathbf{K}^{-}-\mathbf{K}^{\prime-} \mathbf{G}^{-1} \mathbf{T}_{k_{0}}\right),
$$

which can easily be calculated numerically.

For example, consider a cube with side 1 meter, $\epsilon=2 \epsilon_{0}$, $\mu=\mu_{0}$, and $\kappa=0.5$. The surface of the cube is discretized using 1800 expansion functions. The matrix (44) and its eigenvalues are computed for a frequency of $150 \mathrm{MHz}$ (Fig. 4). The eigenvalues accumulate at zero, thus supporting the assertion that $\mathcal{T}_{k_{0}} \mathcal{K}^{-}-\mathcal{K}^{-} \mathcal{T}_{k_{0}}$ is compact.

\section{Application of the PMCHWT Equation to a Chiral Meta- material}

As a last example, the chiral CMP PMCHWT simulation technique is applied to the chiral metamaterial presented in [25]. Chiral particles (Fig. 5) with diameter $2.202 \mathrm{~mm}$ are mixed randomly to create an isotropic chiral metamaterial. The inclusion density is $34.5 \mathrm{~cm}^{-3}$.

When the T-matrix of a spherical ensemble of chiral particles is known, it is possible to derive a closed form expression of the material parameters of an equivalent homogeneous sphere. Using this technique, the authors found that at a
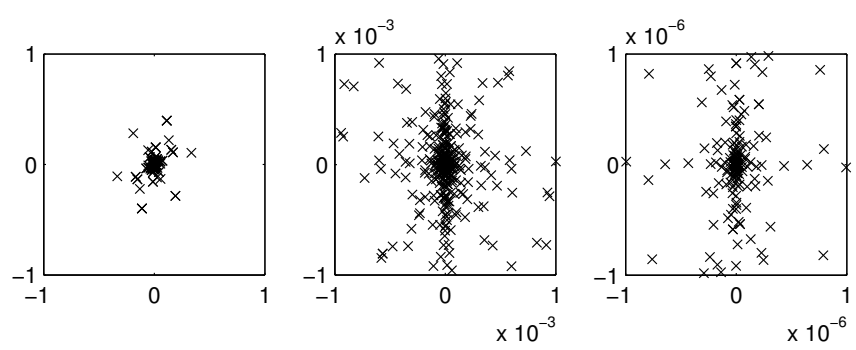

Fig. 4. Location of the eigenvalues of the three-wavenumber operator $\mathcal{T}_{k_{0}} \mathcal{K}^{-}-\mathcal{K}^{-} \mathcal{T}_{k_{0}}$ in the complex plane, applied to a cube. The left panel contains all eigenvalues. The middle and right panel are zoomed in around 0 , where the eigenvalues are seen to accumulate.

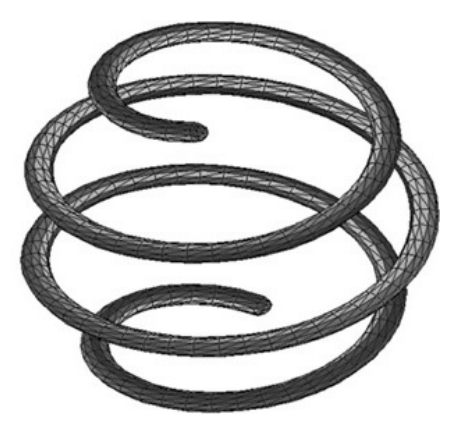

Fig. 5. A chiral particle [25].

frequency of $5.98 \mathrm{GHz}$ (corresponding to a wavelength $\lambda=5$ $\mathrm{cm}$ ), this metamaterial can be described by the following parameters: $\epsilon=1.6347 \epsilon_{0}, \mu=1.1072 \mu_{0}$, and $\kappa=0.1511$.

The bistatic radar cross section of a cuboid of this material with dimensions $10 \mathrm{~cm} \times 10 \mathrm{~cm} \times 5 \mathrm{~cm}$ is computed in the xyplane (Fig. 7). The incoming electric field is linearly polarized along the $\mathrm{z}$ axis and propagates along the directions $\phi^{i n c}=0$ and $\phi^{i n c}=\pi / 4$. The comparison of the condition number and the required number of iterations (averaged over the dipole and the plane wave excitations) with and without CMP again testifies to the success of the CMP (Fig. 8). Without CMP, dense discretization breakdown occurs for mesh parameters $h \leq \lambda / 8$. With CMP, the condition number as well as the required number of iterations remain constant.

Next, the cuboid is excited by two different sources: a dipole located in the symmetry plane of the block, and one residing

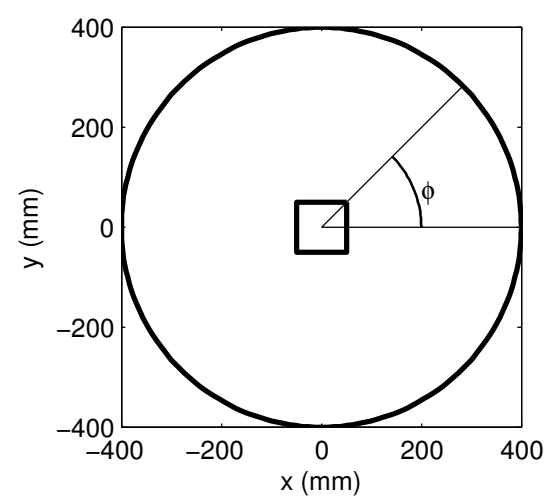

Fig. 6. Definition of the angle $\phi$. 


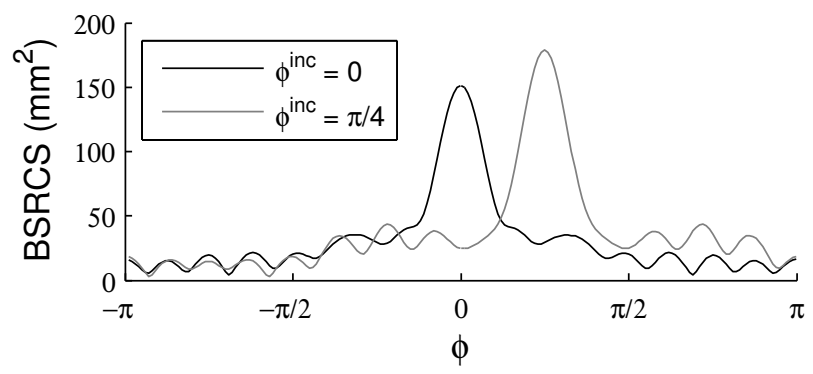

Fig. 7. Bistatic radar cross section in the xy-plane, computed for incoming plane waves propagating along the directions $\phi^{i n c}=0$ and $\phi^{i n c}=\pi / 4$. The angle $\phi$ is defined in Fig. 6 .
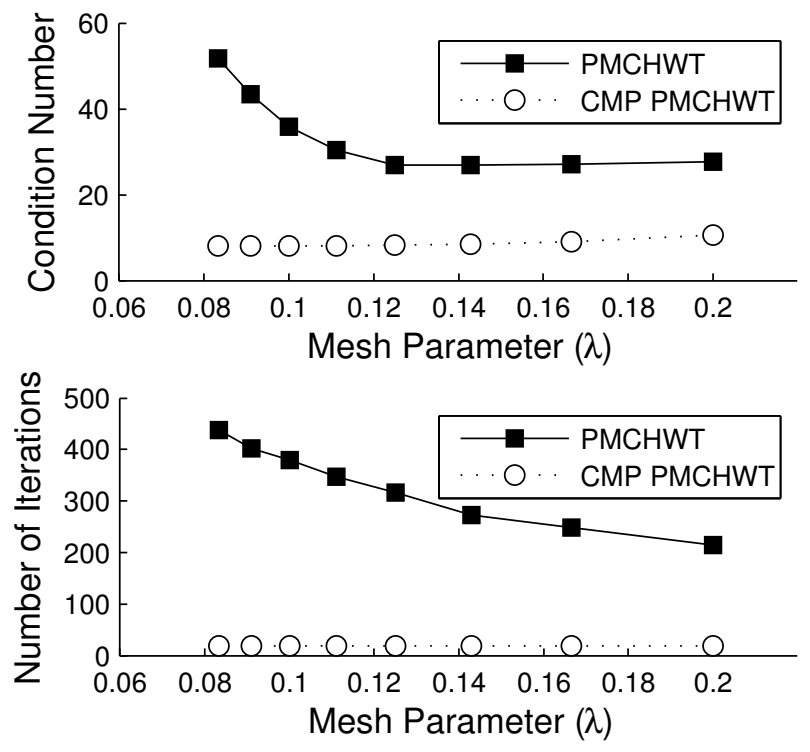

Fig. 8. Condition number of the system matrix (top) and average number of iterations (bottom) for the cuboid, with and without CMP.

in its top plane (Fig. 9). In [25], the field scattered by this configuration was computed "ab initio", that is by accounting for each and every spiral by using the NSPW-MLFMA Tmatrix method. This approach required the solution of a set of linear equations with 347,400 unknowns.

The calculation is now repeated using both the classic chiral PMCHWT and the chiral CMP PMCHWT method by modeling the block as a homogeneous chiral medium, and covering its surface with 2048 expansion functions (the mesh parameter being $\lambda / 8$ ). As is to be expected, the use of the CMP does not alter the results (up to numerical precision). The efficiency of the CMP is once again proven: for the symmetrical excitation, the required number of iterations to reach a relative residual of $10^{-6}$ is reduced from 247 to 16 , and for the asymmetric one from 254 to 17 .

In order to compare the results to the ab initio simulation data provided by the authors of [25], the electromagnetic fields are calculated at a distance of $40 \mathrm{~cm}$ from the center of the block (Fig. 6). The $\boldsymbol{h}_{x}$-component is shown in the top and middle panels of Figs. 10 and 11 for the symmetric and the asymmetric excitations, respectively.

A remark concerning the approximate agreement between

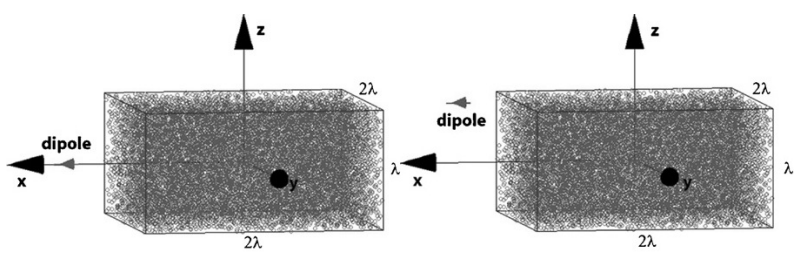

Fig. 9. Position of the dipole source for symmetric (left) and asymmetric (right) excitation [25].

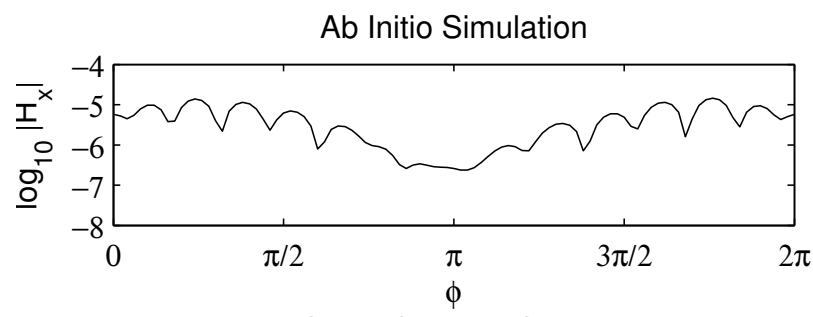

Chiral CMP PMCHWT

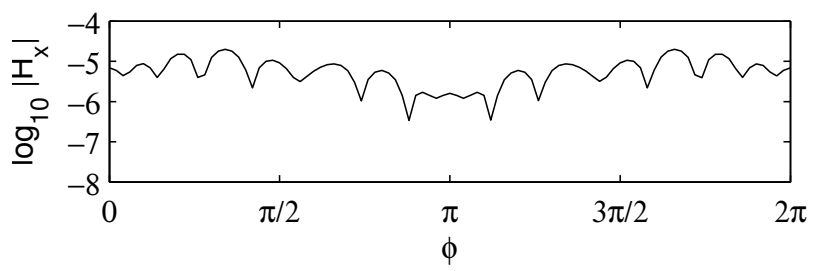

Nonchiral CMP PMCHWT

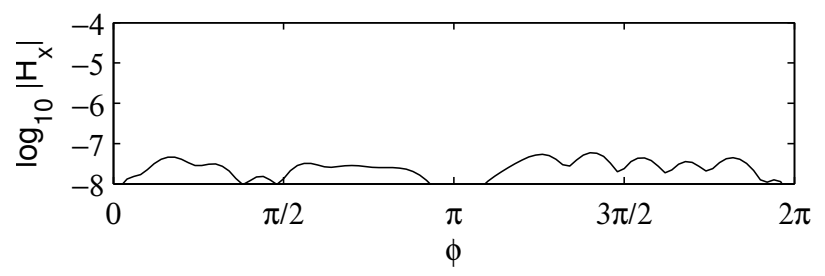

Fig. 10. The scattered field $\log _{10}\left(\left|\boldsymbol{h}_{x}\right|\right)$ due to the symmetric excitation.

Ab Initio Simulation

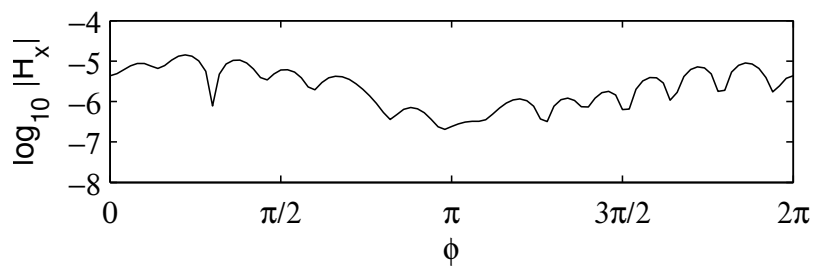

Chiral CMP PMCHWT

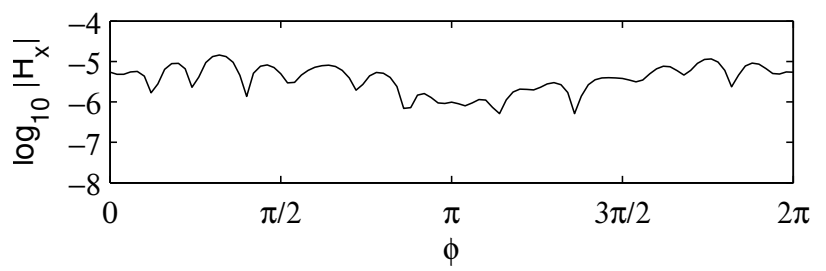

Nonchiral CMP PMCHWT

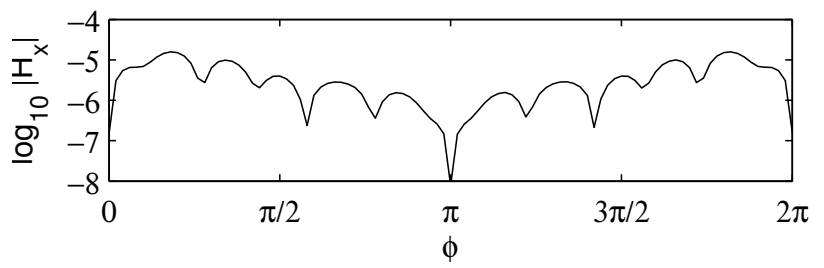

Fig. 11. The scattered field $\log _{10}\left(\left|\boldsymbol{h}_{x}\right|\right)$ due to the asymmetric excitation. 
the results of the ab initio simulation and the chiral PMCHWT method is in order. The constitutive equations (3) describe a homogeneous, continuous medium. However, in this particular example, the microscopic building blocks are relatively large (with dimensions of about $\lambda / 20$ ). Therefore, constitutive equations can only provide an approximate model of the medium. When characterization of the chiral medium by a macroscopic parameter $\kappa$ is warranted, however, the modeling of scattering by such chiral objects obviously can be performed much more efficiently by the chiral CMP PMCHWT than by an ab initio simulation which takes into account the microscopic structure of the material.

The inclusion of a nonzero chirality parameter however does provide a good prediction of the order of magnitude of the different field components. The scattered field computed using the nonchiral PMCHWT method (with the same permittivity and permeability) is shown in the bottom panel of Figs. 10 and 11. For the symmetric excitation, the $\boldsymbol{h}_{x}$-component vanishes (up to the iterative solver's tolerance) for all $\phi$. For the asymmetric excitation, it vanishes at $\phi=0$ and $\phi=\pi$. This is due to incorrect assumptions about the symmetry of the medium. This error is corrected by the inclusion of a nonzero chirality parameter.

\section{CONCLUSIONS}

In this paper, numerical methods for analyzing scattering of electromagnetic fields by chiral media were studied. The main theoretical tool is the Bohren transform, which allows for a far-reaching analogy between chiral media and nonchiral dielectrics. By exploiting this analogy, boundary integral equations for dielectric structures can be extended to chiral objects. The extension of the PMCHWT equation was revisited in detail.

An accurate numerical solution of the chiral PMCHWT equation can be obtained with straightforward discretization schemes using RWG expansion functions. However, as is the case for the nonchiral PMCHWT equation and the EFIE, the condition number of the resulting set of equations quickly grows as the discretization becomes denser; that is, dense discretization breakdown occurs.

By studying two- and three-wavenumber extensions to the Calderón identities used in the Calderón preconditioning of the EFIE for analyzing PEC scattering, it is shown that the square of the chiral PMCHWT operator possesses a bounded spectrum and is therefore not susceptible to dense discretization breakdown. By applying a stable discretization scheme involving both RWG and BC functions to the squared chiral PMCHWT operator, a Calderón multiplicative preconditioner for the chiral PMCHWT equation, which effectively resolves the problem of dense discretization breakdown, was constructed.

Finally, three numerical experiments were performed. First, the accuracy of the chiral PMCHWT method and the efficacy of the CMP were corroborated by comparison of the result they yield, to the analytical solution for scattering by a spherical object. Second, the spectrum of the chiral PMCHWT operator was studied for nonsmooth surfaces. Third, the chiral CMP
PMCHWT method was applied to the analysis of scattering by a metamaterial. These experiments show that the CMP PMCHWT method is able to efficiently and accurately solve scattering problems involving chiral media, provided that these media can be described using constitutive equations, i.e. if they can be considered as homogeneous and isotropic.

\section{REFERENCES}

[1] A. Poggio and E. Miller, Computer Techniques for Electromagnetics. Oxford UK: Pergamon Press, 1973.

[2] C. Müller, Foundations of the Mathematical Theory of Electromagnetic Waves. Springer, 1969.

[3] F. P. Andriulli, K. Cools, H. Bağci, F. Olyslager, A. Buffa, S. Christiansen, and E. Michielssen, "A multiplicative Calderón preconditioner for the electric field integral equation," IEEE Trans. Antennas Propagation, vol. 56, pp. 2398-1930, aug 2008.

[4] K. Cools, F. Andriulli, and E. Michielssen, "A Calderón multiplicative preconditioner for the PMCHWT integral equation," IEEE Trans. Antennas Propag., vol. PP, no. 99, p. 1, 2011.

[5] S. Yan, J.-M. Jin, and Z. Nie, "A comparative study of Calderón preconditioners for PMCHWT equations," IEEE Trans. Antennas Propag. vol. 58, no. 7, pp. $2375-2383$, july 2010.

[6] Y. Beghein, K. Cools, F. Andriulli, D. De Zutter, and E. Michielssen, "Calderon multiplicative preconditioner for the PMCHWT equation applied to chiral media," in Antennas and Propagation (APSURSI), 2011 IEEE International Symposium on, july 2011, pp. 3203 -3206.

[7] H. Ammari, K. Hamdache, and J.-C. Nédélec, "Chirality in the Maxwell equations by the dipole approximation," SIAM J. Appl. Math., vol. 59, pp. 2045-2059, August 1999.

[8] A. Lakhtakia, V. Varadan, and V. Varadan, Time-Harmonic Electromagnetic Fields in Chiral Media. Springer-Verlag, 1989.

[9] D. Colton and R.Kress, Integral Equation Methods in Scattering Theory. Wiley, 1983.

[10] D. Worasawate, J. Mautz, and E. Arvas, "Electromagnetic scattering from an arbitrarily shaped three-dimensional homogeneous chiral body," IEEE Trans. Antennas Propag., vol. 51, no. 5, pp. 1077 - 1084, may 2003.

[11] H. Ammari, J. Nedelec, and H. A. J. C. Nédélec, "Time-harmonic electromagnetic fields in chiral media," 1996.

[12] X. Wang, D. Werner, L.-W. Li, and Y.-B. Gan, "Interaction of electromagnetic waves with 3-d arbitrarily shaped homogeneous chiral targets in the presence of a lossy half space," IEEE Trans. Antennas Propag., vol. 55, no. 12, pp. $3647-3655$, dec. 2007.

[13] R. S. Chen, Y. Q. Hu, Z. H. Fan, D. Z. Ding, D. X. Wang, and E. Yung, "An efficient surface integral equation solution to em scattering by chiral objects above a lossy half space," IEEE Trans. Antennas Propag., vol. 57, no. 11, pp. $3586-3593$, nov. 2009.

[14] C. Athanasiadis, G. Costakis, and I. Stratis, "Electromagnetic scattering by a homogeneous chiral obstacle in a chiral environment," IMA Journal of Applied Mathematics, vol. 64, no. 3, pp. 245-258, 2000.

[15] S. Sauter and C. Schwab, Boundary Element Methods. Springer, 2010.

[16] S. Rao, D. Wilton, and A. Glisson, "Electromagnetic scattering by surfaces of arbitrary shape," IEEE Trans. Antennas Propag., vol. 30, no. 3, pp. $409-418$, may 1982.

[17] H. Contopanagos, B. Dembart, M. Epton, J. Ottusch, V. Rokhlin, J. Visher, and S. Wandzura, "Well-conditioned boundary integral equations for three-dimensional electromagnetic scattering," IEEE Trans. Antennas Propag., vol. 50, pp. 1824-1930, dec 2002.

[18] G. Hanson and A. Yakolev, Operator Theory for Electromagnetics. Springer, 2001.

[19] M. Abramowitz and I. Stegun, Handbook of Mathematical Functions with Formulas, Graphs, and Mathematical Tables, ninth dover printing, tenth gpo printing ed. New York: Dover, 1964.

[20] A. Buffa and S. H. Christiansen, "A dual finite element complex on the barycentric refinement," Comptes Rendus Mathematique, vol. 340, no. 6, pp. $461-464,2005$.

[21] S. Yan, J.-M. Jin, and Z. Nie, "Implementation of the Calderón multiplicative preconditioner for the efie solution with curvilinear triangular patches," in Antennas and Propagation Society International Symposium, 2009. APSURSI '09. IEEE, june 2009, pp. 1 -4

[22] F. Valdes, F. Andriulli, K. Cools, and E. Michielssen, "High-order divand quasi curl-conforming basis functions for Caldern multiplicative preconditioning of the EFIE," IEEE Trans. Antennas Propag., vol. 59, no. 4 , pp. $1321-1337$, april 2011. 
[23] F. Andriulli, F. Valdés, K. Cools, and E. Michielssen, "A generalized Calderón preconditioner for the electric field integral equation," in Antennas and Propagation Society International Symposium (APSURSI), 2010 IEEE, july 2010, pp. $1-4$.

[24] C. F. Bohren, "Light scattering by an optically active sphere," Chemical Physics Letters, vol. 29, no. 3, pp. 458 - 462, 1974

[25] I. Bogaert, J. Peeters, and F. Olyslager, "Homogenization of metamaterials using full-wave simulations," Metamaterials, vol. 2, no. 2-3, pp. 101-112, sep 2008

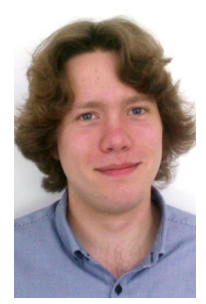

Yves Beghein received the M.Sc.Eng. degree in physical engineering from Ghent University, Belgium, in 2011. His master's dissertation dealt with the efficient modeling of scattering by chiral media. $\mathrm{He}$ is currently pursuing a Ph.D. degree at the Electromagnetics Group at Ghent University, under the advisorship of Prof. Kristof Cools and Prof. Daniël De Zutter. His research focusses on both time domain and frequency domain boundary element methods, their stable and accurate discretizations, and efficient solution methods.

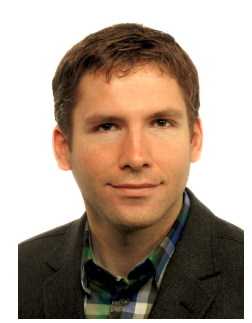

Kristof Cools received the M. Eng. degree in Physical Engineering from Ghent University, Belgium, in 2004. In August 2004, he joined the Electromagnetics Group at Ghent University. He received the Ph.D. degree from Ghent University in 2008, under the advisership of Prof. F. Olyslager and Prof. E. Michielssen. In 2009, he was awarded the Young Scientist Best Paper award at the International Conference on Electromagnetics and Advanced Applications. In 2011, he was a visiting research fellow at TELECOM Bretagne. In November 2011, he took up a position as Lecturer in Computational Electromagnetics at the University of Nottingham. His research focuses on the spectral properties of the boundary integral operators of electromagnetics, on stable and accurate discretization schemes for frequency and time domain boundary element methods, on domain decomposition techniques, and on the implementation of algorithms from computational physics for high performance computing.

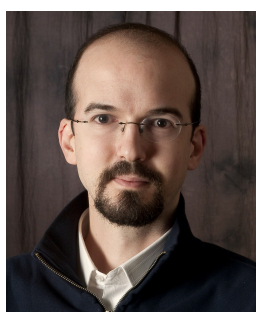

Francesco P. Andriulli (S '05, M '09, SM '11) received the Laurea degree in electrical engineering from the Politecnico di Torino, Italy, in 2004, the M.S. degree in electrical engineering and computer science from the University of Illinois at Chicago in 2004, and the Ph.D. degree in electrical engineering from the University of Michigan at Ann Arbor in 2008. From 2008 to 2010 he was a Research Associate with the Politecnico di Torino. Since 2010 he has been with the Microwave Department of the École nationale supérieure des télécommunications de Bretagne (TELECOM Bretagne), Brest, France, where he currently is a Maître de conférences.

His research interests are in computational electromagnetics with focus on preconditioning and fast solution of frequency and time domain integral equations, integral equation theory, hierarchical techniques, and single source integral equations.

Dr. Andriulli was awarded the University of Michigan International Student Fellowship and the University of Michigan Horace H. Rackham Predoctoral Fellowship. He was the recipient of the best student paper award at the 2007 URSI North American Radio Science Meeting. He received the first place prize of the student paper context of the 2008 IEEE Antennas and Propagation Society International Symposium, where he authored and coauthored other two finalist papers. He was the recipient of the 2009 RMTG Award for junior researchers and was awarded a URSI Young Scientist Award at the 2010 International Symposium on Electromagnetic Theory.

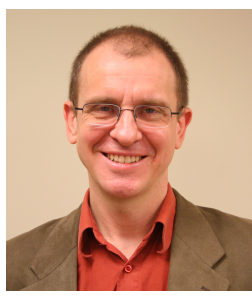

Eric Michielssen received his M.S. in Electrical Engineering (Summa Cum Laude) from the Katholieke Universiteit Leuven (KUL, Belgium) in 1987, and his Ph.D. in Electrical Engineering from the University of Illinois at Urbana-Champaign (UIUC) in 1992. He joined the faculty of the UIUC Department of Electrical and Computer Engineering in 1993, reaching the rank of Full Professor in 2002. In 2005, he joined the University of Michigan (UM) as Professor of Electrical Engineering and Computer Science. Since 2009, he directs the University of Michigan Computational Science Certificate Program.

Eric Michielssen received a Belgian American Educational Foundation Fellowship in 1988 and a Schlumberger Fellowship in 1990. Furthermore, he was the recipient of a 1994 International Union of Radio Scientists (URSI) Young Scientist Fellowship, a 1995 National Science Foundation CAREER Award, and the 1998 Applied Computational Electromagnetics Society (ACES) Valued Service Award. In addition, he was named 1999 URSI United States National Committee Henry G. Booker Fellow and selected as the recipient of the 1999 URSI Koga Gold Medal. He also was awarded the UIUC's 2001 Xerox Award for Faculty Research, appointed 2002 Beckman Fellow in the UIUC Center for Advanced Studies, named 2003 Scholar in the Tel Aviv University Sackler Center for Advanced Studies, selected as UIUC 2003 University and Sony Scholar; in 2011 he received the UM College of Engineering David E. Liddle Research Excellence Award. He is a Fellow of the IEEE (elected 2002) and a member of URSI Commission B.

Eric Michielssen served as the Technical Chairman of the 1997 Applied Computational Electromagnetics Society (ACES) Symposium (Review of Progress in Applied Computational Electromagnetics, March 1997, Monterey, CA), and served on the ACES Board of Directors (1998-2001 and 2002-2003) and as ACES Vice-President (1998-2001). From 1997 to 1999, he was as an Associate Editor for Radio Science, and from 1998 to 2008 he served as Associate Editor for the IEEE Transactions on Antennas and Propagation. Eric Michielssen authored or co-authored over 160 journal papers and book chapters and over 300 papers in conference proceedings. His research interests include all aspects of theoretical and applied computational electromagnetics. His research focuses on the development of fast frequency and time domain integral-equation-based techniques for analyzing electromagnetic phenomena, and the development of robust optimizers for the synthesis of electromagnetic/optical devices.

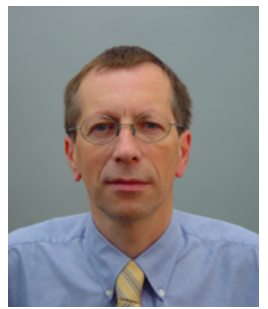

Daniël De Zutter was born in 1953. He received his M. Sc. Degree in electrical engineering from the University of Gent in 1976. In 1981 he obtained a Ph. D. degree and in 1984 he completed a thesis leading to a degree equivalent to the French Aggrégation or the German Habilitation. He is now a full professor of electromagnetics. His research focusses on all aspects of circuit and electromagnetic modelling of high-speed and high-frequency interconnections and packaging, on Electromagnetic Compatibility (EMC) and numerical solutions of Maxwells equations. As author or co-author he has contributed to more than 180 international journal papers (cited in the Web of Science) and 200 papers in conference proceedings. In 2000 he was elected to the grade of Fellow of the IEEE. He was an Associate Editor for the IEEE Microwave Theory and Techniques Transactions. Between 2004 and 2008 he served as the Dean of the Faculty of Engineering of Ghent University and is now the head of the Department of Information Technology. 